\title{
Improved Frontoparietal White Matter Integrity in Overweight Children Is Associated with Attendance at an After-School Exercise Program
}

\author{
Cynthia E. Krafft ${ }^{a}$ David J. Schaeffer ${ }^{b} \quad$ Nicolette F. Schwarz ${ }^{a} \quad$ Lingxi Chi $^{a}$ \\ Abby L. Weinberger ${ }^{a}$ Jordan E. Pierce ${ }^{a}$ Amanda L. Rodrigue ${ }^{a}$ \\ Jerry D. Allison ${ }^{d}$ Nathan E. Yanasak ${ }^{d}$ Tianming Liu ${ }^{c}$ Catherine L. Davis $^{e, f}$ \\ Jennifer E. McDowell ${ }^{a}$ \\ Departments of a Psychology, ${ }^{b}$ Neuroscience and ${ }^{c}$ Computer Science, University of Georgia, Athens, Ga., \\ Departments of ${ }^{d}$ Radiology and ${ }^{e}$ Pediatrics, and ${ }^{f}$ Georgia Prevention Center, Medical College of Georgia, \\ Georgia Regents University, Augusta, Ga., USA
}

\section{Key Words}

Aerobic exercise - Children - Cognition - Cognitive control .

Diffusion tensor imaging $\cdot$ Overweight $\cdot$ Superior

longitudinal fasciculus

\begin{abstract}
Aerobic fitness is associated with white matter integrity (WMI) in adults as measured by diffusion tensor imaging (DTI). This study examined the effect of an 8-month exercise intervention on WMI in children. Participants were 18 sedentary, overweight (BMI $\geq 85$ th percentile) 8- to 11-year-old children (94\% Black), randomly assigned to either an aerobic exercise $(n=10)$ or sedentary attention control group $(n=$ 8). Each group was offered an instructor-led after-school program every school day for approximately 8 months. Before and after the program, all subjects participated in DTI scans. Tractography was conducted to isolate the superior longitudinal fasciculus and investigate whether the exercise intervention affected WMI in this region. There was no group by time interaction for WMI in the superior longitudinal fasciculus. There was a group by time by attendance interaction, however, such that higher attendance at the exercise intervention, but not the control intervention, was associ-
\end{abstract}

\section{KARGER}

E-Mail karger@karger.com

www.karger.com/dne ated with increased WMI. Heart rate and the total dose of exercise correlated with WMI changes in the exercise group. In the overall sample, increased WMI was associated with improved scores on a measure of attention and improved teacher ratings of executive function. This study indicates that participating in an exercise intervention improves WMI in children as compared to a sedentary after-school program.

(c) 2014 S. Karger AG, Basel

\section{Introduction}

Aerobic fitness is associated with cognitive performance across the life-span. In cross-sectional studies, higher-fit individuals showed better performance than lower-fit peers on measures of cognitive control, and higher-fit children demonstrated comparatively better academic achievement and parent and teacher ratings of behavior [1-5]. There is evidence that aerobic exercise plays a causal role

Co-corresponding author: Catherine L. Davis

Georgia Prevention Center, Institute of Public and Preventive Health

Medical College of Georgia, Georgia Regents University

Augusta, GA 30912 (USA)

E-Mail cadavis@gru.edu

Jennifer E. McDowell

Department of Psychology

University of Georgia

Athens, GA 30602 (USA)

E-Mail jemcd@uga.edu 
in improving cognition, as aerobic exercise training improves performance on a variety of cognitive tasks. Randomized controlled trials showed that both adults and children who were assigned to chronic exercise improved cognitive performance compared to their peers assigned to control groups [6-8], with more improvement associated with greater attendance [9]. While exercise benefits many types of cognition, there is evidence that exercise-related improvement is greater for cognitive control (higher-order processes including inhibition, working memory, and attentional control) compared to lower-level tasks [10].

Cognitive control is supported by frontoparietal circuitry $[11,12]$. Exercise interventions improved cognitive control and altered frontal and parietal activation as assessed by functional MRI. Specifically, a 6-month exercise intervention in older adults improved performance and increased prefrontal and posterior parietal activation during a flanker task in an exercise group as compared to controls [7]. Regional alterations were also found in studies of children. Our group initially found that a 3-month exercise intervention in 7- to 11-year-old children improved cognitive control performance [as measured by the Planning scale of the Cognitive Assessment System (CAS)] compared to controls [6]. The exercise group also increased prefrontal and decreased posterior parietal activation during an antisaccade task compared to controls. We recently extended these findings using an 8-month intervention (the current study). We found that children assigned to 8 months of exercise showed altered activation during antisaccade and flanker tasks as compared to the sedentary attention control group [13].

With evidence that frontoparietal brain activation is affected by exercise, one issue that warrants investigation is whether exercise alters frontoparietal brain structure. Altered white matter structure may be an underlying cause of functional change, given evidence that inter-individual differences in brain activation reflect white matter integrity (WMI) [14]. WMI reflects axonal membrane structure and myelination and can be assessed by diffusion tensor imaging (DTI), which measures the anisotropy (directional dependence) of water diffusion. Fractional anisotropy (FA) is a frequent measure of interest in DTI and describes the anisotropy of water diffusion. FA values range between 0 and 1 , with 1 indicating fully anisotropic diffusion. Higher values are generally interpreted as greater WMI (myelination and axonal membrane structure [15]). Another measure based on the same tensor model is radial diffusivity (RD), which measures water diffusion perpendicular to axons (with higher values indicating greater diffusion). Taken together, higher FA and lower RD values are often interpreted as primarily reflecting greater myelination $[16,17]$.

WMI was associated with fitness in several cross-sectional studies. Higher aerobic fitness in adults was associated with higher FA in the cingulum and corpus callosum, possibly relating to motor planning and control [18-20]. Fitness was also associated with integrity of the uncinate fasciculus, which is involved in memory [20]. In one of the first investigations to date on the impact of a randomized controlled exercise intervention on WMI, Voss et al. [21] randomly assigned adults to 1 year of either an aerobic exercise group or a flexibility and toning control group. Increased aerobic fitness was associated with increases in prefrontal, parietal, and temporal FA in the aerobic group. While the literature indicates that exercise affects WMI in adults, this topic has yet to be investigated in children.

Given evidence that exercise improved cognitive control and altered associated frontoparietal brain activation in prior studies, we investigated whether an exercise intervention in overweight children improves WMI in a tract that connects frontal and parietal regions: the superior longitudinal fasciculus (SLF). Only overweight participants were recruited for the current study since sedentary, obese children are likely to show the greatest benefits from exercise. Greater integrity of the SLF is related to better performance on several measures of cognitive control, such as working memory and attention $[16,22,23]$. As the SLF does not completely mature until young adulthood [24], ongoing development makes it an interesting target for investigation across the ages included in the current study (children 8-11 years old). The SLF may be particularly susceptible to intervention effects due to its continuing maturation; it was altered by another type of intervention in children. Five weeks of spelling training decreased RD in the right SLF in children 9-16 years of age [25]. In sum, the hypotheses were generated based on the literature indicating that exercise improves cognitive control, and that fitness is associated cross-sectionally with higher WMI. Specifically, we hypothesized that a randomized controlled exercise intervention in children would increase WMI in the SLF, particularly in children with good attendance.

\section{Methods and Procedures}

\section{Participants}

Participants (94\% Black) were a subset of children in a larger randomized trial [13] recruited from public schools around Augusta, Ga., USA. They were eligible if they were 8-11 years old, overweight (BMI $\geq 85$ th percentile [26]), and inactive (no regular 
Table 1. Baseline characteristics and attendance of participants included in the analysis

\begin{tabular}{lccc}
\hline Characteristic & $\begin{array}{l}\text { Exercise group } \\
(\mathrm{n}=10)\end{array}$ & $\begin{array}{l}\text { Control group } \\
(\mathrm{n}=8)\end{array}$ & $\mathrm{p}$ value \\
\hline Age, years & $9.9 \pm 0.6$ & $9.4 \pm 0.8$ & $0.17^{\mathrm{a}}$ \\
Female, \% & 50 & 50 & $1^{\mathrm{b}}$ \\
Non-White, \% & 100 & 88 & $0.25^{\mathrm{b}}$ \\
Left-handed, \% & 10 & 25 & $0.40^{\mathrm{b}}$ \\
Parental education scale & $4.8 \pm 1.0$ & $4.3 \pm 1.5$ & $0.37^{\mathrm{a}}$ \\
CAS Full Scale score & $94.2 \pm 8.9$ & $95.6 \pm 9.5$ & $0.75^{\mathrm{a}}$ \\
CAS Planning Scale score & $88.2 \pm 11.7$ & $89.5 \pm 13.9$ & $0.83^{\mathrm{a}}$ \\
CAS Attention Scale score & $98.4 \pm 8.5$ & $94.8 \pm 7.5$ & $0.35^{\mathrm{a}}$ \\
BRIEF Global Executive Composite score & $56.0 \pm 11.8$ & $47.9 \pm 9.8$ & $0.16^{\mathrm{a}}$ \\
BRIEF Behavioral Regulation Index score & $54.3 \pm 11.4$ & $45.6 \pm 3.7$ & $0.07^{\mathrm{a}}$ \\
BRIEF Metacognition Index score & $55.9 \pm 12.0$ & $49.1 \pm 12.4$ & $0.27^{\mathrm{a}}$ \\
Attendance, \% & $60 \pm 10$ & $72 \pm 8$ & $0.36^{\mathrm{a}}$ \\
\hline
\end{tabular}

Values are presented as mean \pm SD or percentages unless indicated otherwise. ${ }^{a} t$ test. ${ }^{b} \chi^{2}$ test.

physical activity program $\geq 1 \mathrm{~h}$ /week). Exclusions included any medical condition that would limit physical activity or affect study results (including neurological or psychiatric disorders). Children and parents completed written informed assent and consent forms in accordance with the Human Assurance Committee of Georgia Regents University. Each child's parent or guardian reported the child's age, sex, race, and health status. Parents also reported their own educational attainment, used as an index of socioeconomic status ( $1=7$ or fewer years of school; $2=8-9$ years of school; $3=$ $10-11$ years of school; $4=12$ years of school; 5 = partial college; $6=$ college graduate, and $7=$ postgraduate). The study took place at the Georgia Prevention Center at Georgia Regents University. MRI was completed with DTI data available for 41 children at baseline and 30 at posttest. Three of the 30 children scanned at posttest had not been scanned at baseline. Of the 27 children with both baseline and posttest data, 8 were excluded due to scanner artifact or excessive motion, and 1 was excluded due to a neurological anomaly. Thus, the present study included 18 children (10 exercise, 8 control; see table 1 ).

\section{Cognitive Measures}

The CAS, a standardized individual assessment of children's cognitive processes [27], was administered, and standard scores were analyzed for all 18 children at both baseline and posttest. The Full Scale score of the CAS takes into account the 4 scales of the test (Planning, Attention, Simultaneous, and Successive Processing), each of which include 3 subtests. For the CAS, higher scores reflect better performance. The Behavioral Rating Inventory of Executive Function (BRIEF) was completed by up to 3 teachers per child [28]. This questionnaire provides a standardized measure of executive function behaviors in the school environment, with higher scores reflecting worse performance. For each child, teachers completing the BRIEF were matched at baseline and posttest to maintain consistency. $\mathrm{t}$ scores were obtained for 17 children at baseline (10 exercise, 7 control) and 16 at posttest ( 9 exercise, 7 control), with 15 children having both baseline and posttest data and thus being included in BRIEF analyses ( 9 exercise, 6 control). The BRIEF was completed by an average of 1.6 teachers $(\mathrm{SD}=0.8)$ per child, with no significant difference in the number of teachers between groups $(t(13)=1.02, \mathrm{p}>0.3)$.

\section{Intervention}

Participants were randomly assigned to 1 of 2 conditions: aerobic exercise or sedentary attention control. Randomization (balanced by race, sex, and school) was performed by the study statistician and concealed until after baseline testing was completed, at which point the study coordinator informed the families. Both groups were offered an after-school program every school day for approximately 8 months (average number of days offered $=139$, $\mathrm{SD}=9$ ). All participants were offered daily bus transportation after school to the Georgia Prevention Center where they spent half an hour on supervised homework time and were given a snack. Lead instructors were rotated between the 2 groups every 2 weeks and assistants were rotated between the 2 groups every week.

The aerobic exercise group engaged in instructor-led aerobic activities (e.g. tag and jump rope) for $40 \mathrm{~min}$ per day. They wore heart rate monitors every day (S610i; Polar Electro, Oy, Finland) with which they could monitor their own performance and from which data were collected daily. Participants in the exercise group had an average heart rate of 161 beats per minute $(S D=8)$ during the intervention. The product of each child's average heart rate and their attendance provided an index of total energy expenditure during the exercise intervention. The attention control group engaged in instructor-led sedentary activities (e.g. art and board games).

\section{DTI Procedure and Analysis}

MRI Acquisition

Images were acquired at Georgia Regents University on a 3T GE Signa Excite HDx MRI system (General Electric Medical Sys- 
tems, Milwaukee, Wis., USA). During scanning, head position was stabilized with a vacuum pillow and/or foam padding. Diffusion images were acquired using an echo planar imaging sequence (acquisition matrix $=128 \times 128,60$ interleaved slices, voxel size $=1 \times 1 \times 2.4 \mathrm{~mm}, \mathrm{FOV}=256 \times 256 \mathrm{~mm}, \mathrm{TR}=15,500$ $\mathrm{ms}$, TE $=$ min-full, 3 B0 images, 30 diffusion-weighted images, $\left.\mathrm{b}=1,000 \mathrm{~s} / \mathrm{mm}^{2}\right)$.

\section{Image Analysis}

Raw diffusion images were converted from GE DICOM format to NIfTI format using the dcm2nii tool [29]. For each subject, volumes were visually inspected for motion artifacts; volumes distorted by motion were removed from the image series and $\mathrm{b}$ value/vector tables (mean volumes removed $=2, \mathrm{SD}=2.7$ ). To test for inhomogeneity of gradient application due to volume removal, volume gradient vectors were plotted on a sphere after motion volumes were removed. If any surface of the sphere had a gap greater than the free surface area of 6 noncollinear directions, the participant was excluded from analysis (2 participants were thus excluded for excessive motion). Diffusion tensor image preprocessing was conducted using the FMRIB software library [30]. Nonbrain tissue was removed using the brain extraction tool [31]. Diffusion images were corrected for eddy-current-induced distortions. Tractography was then conducted with wholebrain tensors in individual space for the bilateral SLF following established anatomical markers [32] using the ExploreDTI software package [33]. Average values for each SLF (right and left) were extracted for 4 measures of white matter microstructure, all of which were included in analyses: FA, RD, mean diffusivity $(\mathrm{MD})$, and axial diffusivity (AD). High $\mathrm{MD}$ values are often explained as disorganized development, immaturity, or other structural anomalies in white matter, whereas high $\mathrm{AD}$ values are thought to reflect a lower number of axonal fibers or smaller axonal diameter.

Statistical analyses of white matter variables were conducted using SPSS Version 21 (IBM, Armonk, N.Y., USA). Repeated measures ANOVAs were conducted for each measure of WMI to examine effects of group, attendance, measurement time, and their interactions on the outcome. Each model controlled for age and gender. Group by time interactions were the primary tests of our hypothesis. Time by group by attendance interactions tested whether attendance was associated with change in WMI differently between groups. The same analyses also were conducted for cognitive measures. Correlations between change in WMI and attendance were conducted separately for each group to examine significant group by attendance interactions. For the exercise group alone, correlations were conducted between change in WMI and average heart rate at the intervention as well as the product of average heart rate and attendance.

An exploratory analysis was conducted to investigate whether improved WMI was associated with improved cognitive performance in the overall sample. For each white matter variable that showed a significant time by group by attendance interaction, the change from baseline to posttest was calculated for each individual, controlling for age and gender. These difference scores were then entered into 2 separate $\mathrm{k}$-means clustering analyses, 1 each for the left and right SLF ( $k=2$, input: FA and RD for the hemisphere of interest; $\leq 10$ iterations). $t$ tests evaluated differences in cognitive task performance between the resulting clusters.

\section{Results}

\section{Cognitive Measures}

Participants attended an average of 3.3 days per week (mean attendance $=65 \%, \mathrm{SD}=6 \%$ ) and the groups did not differ significantly in the percentage of days they attended the program out of the number of days offered $(\mathrm{t}(16)=0.95, \mathrm{p}=0.36)$. The groups did not differ significantly at baseline on any of the characteristics listed in table 1 . There was no significant group by time interaction in any cognitive measure, indicating that the exercise intervention did not differentially affect cognition compared to the control condition. Time by group by attendance interactions were significant for BRIEF Global Executive Composite and Metacognition Index scores, $\mathrm{F}(1,9)=13.17, \mathrm{p}<0.01$ and $\mathrm{F}(1,9)=8.49, \mathrm{p}=0.02$, respectively. For both interactions, attendance at the exercise intervention was associated with more improvement in scores as compared to attendance at the control group session. Partial correlations between attendance and change in BRIEF scores (controlling for age and gender) were not significant for either group alone (a trend level correlation was found between attendance and worse Metacognition Index scores in the control group, $\mathrm{r}(2)=$ $0.94, \mathrm{p}=0.06)$.

\section{White Matter Structure}

There was no significant group by time interaction in any WMI measure, indicating that the exercise intervention did not differentially affect WMI compared to the control condition. Time by group by attendance interactions were significant in the left SLF for both FA $(F(1,12)$ $=7.4, \mathrm{p}=0.02)$ and $\mathrm{RD}(\mathrm{F}(1,12)=5.3, \mathrm{p}=0.04)$, in the right SLF for $\mathrm{RD}(\mathrm{F}(1,12)=5.4, \mathrm{p}=0.04)$, and at the trend level for $\mathrm{FA}(\mathrm{F}(1,12)=4.2, \mathrm{p}=0.06)$. For all group by time by attendance interactions, the exercise group showed a significant relationship between attendance and change in WMI, with increased WMI associated with more frequent attendance $(\mathrm{r}(6)=0.92, \mathrm{p}<0.01$ for right SLF FA and $\mathrm{r}(6)=-0.88, \mathrm{p}<0.01$ for right SLF RD) or trend level $(r(6)=0.64, p=0.06$ for left SLF FA and $r(6)=-0.69$, $\mathrm{p}=0.06$ for left SLF RD). The control group did not show significant relationships between attendance and change in WMI (all p values $>0.10$ ). Group by attendance interactions were not significant for MD or AD. For further details, see figure 1 .

In addition to attendance, the intensity and dose of exercise were related to improved WMI in the exercise group. Higher average heart rate in the exercise intervention was correlated with decreased $\mathrm{MD}(\mathrm{r}(6)=-0.71, \mathrm{p}<$ 


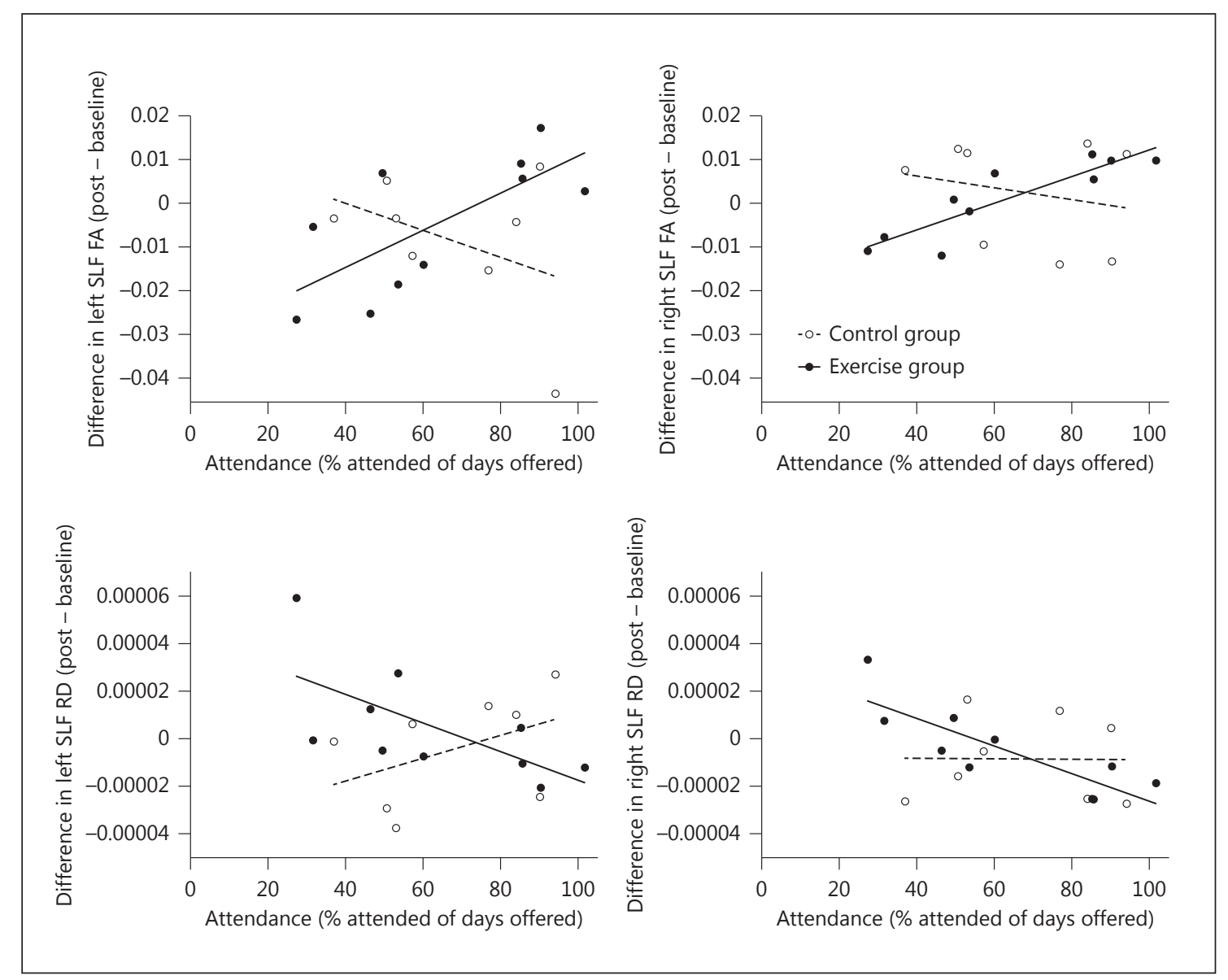

Fig. 1. Time by group by attendance interactions. y-axes show white matter difference scores (changes in integrity from baseline to posttest) and x-axes show attendance. Each measure shown is a residual (controlled for age and gender), which has been back-transformed to its original metric by adding its mean.

$0.05)$ and $\mathrm{RD}(\mathrm{r}(6)=-0.75, \mathrm{p}<0.05)$ in the left SLF. A measure of the dose of exercise (the product of average heart rate and attendance) was correlated with increased FA in the left and right SLF $(r(6)=0.73, p=0.04$ and $\mathrm{r}(6)$ $=0.93, \mathrm{p}<0.01$, respectively), decreased MD in the right SLF $(r(6)=-0.75, p=0.03)$, and decreased RD in the left and right $\operatorname{SLF}(\mathrm{r}(6)=-0.74, \mathrm{p}=0.04$ and $\mathrm{r}(6)=-0.87, \mathrm{p}<$ 0.01 , respectively). There were no significant correlations between age and any measure of WMI at either baseline or posttest (all p values $>0.07$ ).

\section{Exploratory k-Means Analyses}

Cluster analyses were conducted to supplement the main results by illustrating the overall relationship between change in WMI and change in cognitive performance. Analyses divided all participants into 2 groups based on those who improved WMI (FA and RD) and those who did not for each SLF (left and right). With 2 groups identified for each SLF, it was then possible to compare alterations in cognitive measures (CAS and BRIEF scores) between the improved and not-improved SLF groups in order to investigate whether improved WMI was associated with improved cognition in the overall study.

\section{Cluster Analysis 1: Left SLF}

Two clusters were identified which significantly differed in the change in WMI from baseline to posttest. Cluster membership did not significantly overlap with exercise/control group membership. The cluster that showed greater improvement in WMI also showed better cognitive outcome as indicated by decreased BRIEF scores. Spe- 


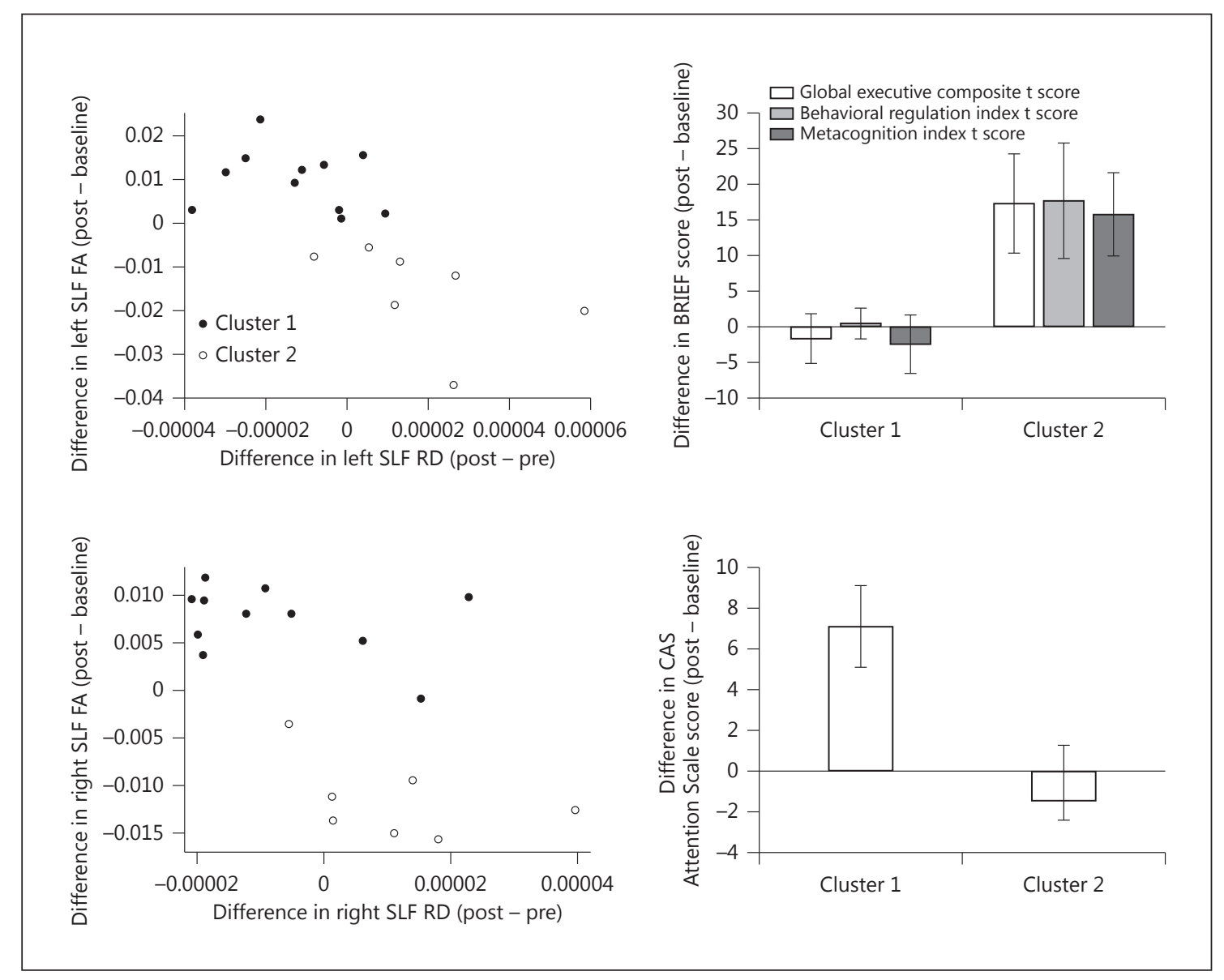

Fig. 2. Illustrations of k-means cluster analyses. The scatterplots illustrate the 2 clusters identified for each analysis based on the participants' alterations in WMI (FA and RD) over time. These show that for both analyses, cluster 1 had improved WMI (increased FA and decreased RD) compared to cluster 2. The bar graphs illustrate the differences in cognition between the 2 clusters obtained in each analysis. In cluster analysis 1, cluster 2 showed worse teacher ratings of executive function as compared to cluster 1 , indicated by increased BRIEF scores. In cluster analysis 2 , cluster 1 showed improved attention scores as compared to cluster 2, indicated by increased CAS Attention Scale scores. The 2 clusters obtained in each k-means analysis significantly differed in all measures shown. Measures shown are difference scores (change from baseline to posttest). cifically, cluster $1(\mathrm{n}=11)$ was composed of participants with improved left SLF WMI as indicated by increased FA $(\mathrm{t}(16)=-6.1, \mathrm{p}<0.01)$ and decreased $\mathrm{RD}(\mathrm{t}(16)=3.7, \mathrm{p}<$ $0.01)$ as compared to cluster $2(\mathrm{n}=7)$. Cluster 1 was associated with more cognitive improvement, significantly differing from cluster 2 in change in BRIEF scores over time. Cluster 2 had significantly worse scores on the BRIEF as compared to cluster 1, including the Global Executive Composite score $(\mathrm{t}(13)=3.2, \mathrm{p}<0.01)$ as well as both the Behavioral Regulation and Metacognition indices $(t(13)=2.5, p=0.04$ and $t(13)=3.04, p=0.01$, respectively). No differences were observed between the 2 clusters in CAS scores. For further details, see figure 2.

\section{Cluster Analysis 2: Right SLF}

Two clusters were identified which significantly differed in the change in WMI from baseline to posttest. Cluster membership did not significantly overlap with exercise/control group membership. The cluster showing greater improvement in WMI also showed better cognitive outcome as indicated by increased CAS Attention Scale scores. Specifically, cluster $1(\mathrm{n}=11)$ was composed of participants with improved right SLF WMI as indicated by increased FA $(\mathrm{t}(16)=10, \mathrm{p}<0.01)$ and decreased $\mathrm{RD}(\mathrm{t}(16)=2.5, \mathrm{p}=0.02)$ as compared to cluster $2(\mathrm{n}=$ 7). Cluster 1 was associated with more cognitive improvement, significantly differing from cluster 2 in changes 
in attention scores over time. Cluster 1 significantly improved scores on the CAS Attention Scale as compared to cluster $2(\mathrm{t}(16)=2.6, \mathrm{p}=0.02)$. No differences were observed between the 2 clusters in BRIEF scores. While both cluster analyses (cluster analysis 1 and cluster analysis 2) did identify similar groups of participants as indicated by a $\chi^{2}$ test $\left(\chi^{2}(1, \mathrm{n}=18)=5.1, \mathrm{p}=0.02\right)$, they did not completely overlap with one another, with a total of 4 participants having different cluster membership between the 2 analyses. For further details, see figure 2.

\section{Discussion}

The current study hypothesized that SLF WMI would be improved by an exercise intervention in overweight children, particularly with greater attendance. This hypothesis was based on evidence that frontoparietal regions and the cognitive control processes that they support are altered by exercise. While no group by time interaction for the SLF was detected, a group by time by attendance interaction indicated that improved WMI in the SLF of overweight children was associated with attendance at an after-school exercise program as compared to attendance at an after-school sedentary program. Specifically, higher attendance at the exercise program was associated with increased WMI in the bilateral SLF (increased FA and decreased RD) as compared to the control group, where attendance was not associated with change in WMI. A dose-response gradient was observed not only with attendance but also with aerobic intensity (heart rate) and an index of total energy expenditure (heart rate $x$ attendance) in the exercise group, such that better attendance, higher intensity, and greater total dose of exercise were linked with greater improvements in SLF WMI. In addition, attendance at the exercise intervention as compared to attendance at the control group session was associated with more improved teacher ratings of cognitive control as measured by the BRIEF. Finally, these results indicated that in the overall study sample, increasing WMI in the SLF benefited cognitive control. Improved WMI in the right SLF was associated with improved selective attention, while improved WMI in the left SLF was associated with better teacher ratings of classroom behavior.

Improved WMI across both groups was associated with better teacher ratings of executive function behaviors in the classroom as assessed by the BRIEF. WMI of the left SLF was associated with a global assessment of executive function and with 2 component scales. Behav-

Exercise Attendance and White Matter ioral Regulation includes items such as children's ability to control impulses and move freely from one situation to the next. Metacognition includes items such as children's ability to initiate activities, hold information in working memory, plan and organize future events, and monitor their own performance. The association between increased WMI and improved teacher ratings indicates that the SLF is important for cognitive control abilities in this sample, as shown in previous studies [22,23]. It also indicates that WMI is reflected in classroom conduct and that it therefore may have implications for academic achievement and social behavior.

The other measure which was associated with WMI across both groups was the CAS Attention Scale, a composite measure based on 3 selective attention tasks: a variation of the Stroop test and 2 paper-and-pencil tests requiring children to find visual targets within fields of distracters. There is previous evidence to suggest that the SLF is important in attention. FA in the SLF has been associated with similar measures in children, such as the sensitivity index of a rapid visual information-processing paradigm, which describes a subject's ability to detect signals independent of answering strategy or bias [34]. SLF FA also was related to visual search in adults (i.e. finding targets among distracters [16]).

Higher FA is often interpreted as reflecting coherently bundled, myelinated fibers, but it is somewhat nonspecific in that it can be affected by various tissue characteristics, including myelination, axon diameter, and fiber organization [35]. It is possible that decreasing $\mathrm{RD}$ in the current study could reflect increasing myelination more so than other alterations in axonal structure, for which $\mathrm{AD}$ might be a more sensitive measure $[17,36]$. Therefore, the changes in WMI described in the current study may be more strongly related to myelination. This interpretation is made with caution, as the association of white matter measures with specific tissue characteristics remains a topic of debate [37]. Nevertheless, the current study indicates that FA and $\mathrm{RD}$ are affected by exercise rather than $\mathrm{AD}$, which is supported by a previous crosssectional study in adults showing that higher FA associated with fitness was primarily related to less $\mathrm{RD}$, rather than altered AD [18]. While this study is one of the first to investigate how exercise affects white matter structure in children, it is limited by a small sample size. This limited power to detect group by time effects and may be susceptible to sample-specific results.

The current study suggests that children's attendance at an after-school exercise program, as compared to a sedentary program, improves WMI in a tract supporting 
cognitive control processes as well as teacher ratings of children's cognitive control behaviors in the classroom. This sample is unique among neuroimaging investigations of exercise in children due to the stringent nature of the control group. Because both groups attended afterschool programs, several potentially beneficial effects were controlled for (e.g. social interaction, attention from adults, and supervised homework time). Additional strengths of this study include the 8-month length of the interventions and the predominantly minority sample of children. Across both groups, improved WMI was related to 2 different measures of cognitive control - a measure of selective attention and teacher ratings of classroom behavior. Increased integrity of the SLF with attendance at an exercise intervention may contribute to improved cognitive control, with potential benefits not only for performance on a standardized cognitive task but also for ontask behavior and interpersonal interactions in the classroom. This may be important to protect school resources for physical activity in the context of unprecedented levels of childhood obesity along with pressure on schools to improve achievement $[38,39]$.

\section{Acknowledgements}

This research was supported by the National Institutes of Health (R01 HL87923) and the National Science Foundation Graduate Research Fellowship Program.

\section{References}

$>1$ Davis CL, Cooper S: Fitness, fatness, cognition, behavior, and academic achievement among overweight children: do cross-sectional associations correspond to exercise trial outcomes? Prev Med 2011;52:S65-S69.

-2 Voss MW, Chaddock L, Kim JS, VanPatter M, Pontifex MB, Raine LB, Cohen NJ, Hillman $\mathrm{CH}$, Kramer AF: Aerobic fitness is associated with greater efficiency of the network underlying cognitive control in preadolescent children. Neuroscience 2011;199:166-176.

-3 Weinstein AM, Voss MW, Prakash RS, Chaddock L, Szabo A, White SM, Wojcicki TR, Mailey E, McAuley E, Kramer AF, Erickson $\mathrm{KI}$ : The association between aerobic fitness and executive function is mediated by prefrontal cortex volume. Brain Behav Immun 2012;26:811-819.

4 Wu CT, Pontifex MB, Raine LB, Chaddock L, Voss MW, Kramer AF, Hillman CH: Aerobic fitness and response variability in preadolescent children performing a cognitive control task. Neuropsychology 2011;25:333-341.

5 Chaddock L, Erickson KI, Prakash RS, VanPatter M, Voss MW, Pontifex MB, Raine LB, Hillman $\mathrm{CH}$, Kramer AF: Basal ganglia volume is associated with aerobic fitness in preadolescent children. Dev Neurosci 2010;32: 249-256.

6 Davis CL, Tomporowski PD, McDowell JE, Austin BP, Miller PH, Yanasak NE, Allison JD, Naglieri JA: Exercise improves executive function and achievement and alters brain activation in overweight children: a randomized controlled trial. Health Psychol 2011;30:9198.

7 Colcombe SJ, Kramer AF, Erickson KI, Scalf P, McAuley E, Cohen NJ, Webb A, Jerome GJ, Marquez DX, Elavsky S: Cardiovascular fitness, cortical plasticity, and aging. Proc Natl Acad Sci USA 2004;101:3316-3321.
$>8$ Kamijo K, Pontifex MB, O’Leary KC, Scudder MR, Wu CT, Castelli DM, Hillman CH: The effects of an afterschool physical activity program on working memory in preadolescent children. Dev Sci 2011;14:1046-1058.

$>9$ Evers A, Klusmann V, Schwarzer R, Heuser I: Improving cognition by adherence to physical or mental exercise: a moderated mediation analysis. Aging Ment Health 2011;15:446-455.

10 Colcombe SJ, Kramer AF: Fitness effects on the cognitive function of older adults: a metaanalytic study. Psychol Sci 2003;14:125-130.

11 Luna B, Thulborn KR, Munoz DP, Merriam EP, Garver KE, Minshew NJ, Keshavan MS, Genovese CR, Eddy WF, Sweeney JA: Maturation of widely distributed brain function subserves cognitive development. Neuroimage 2001;13:786-793.

12 Schaeffer DJ, Amlung MT, Li Q, Krafft CE, Austin BP, Dyckman KA, McDowell JE: Neural correlates of behavioral variation in healthy adults' antisaccade performance. Psychophysiology 2013;50:325-333.

$>13$ Krafft CE, Schwarz NF, Chi L, Weinberger AL, Schaeffer DJ, Pierce JE, Rodrigue AL, Yanasak NE, Miller PH, Tomporowski PD, Davis CL, McDowell JE: An eight month randomized controlled exercise trial alters brain activation during cognitive tasks in overweight children. Obesity 2014;22:232-242.

14 Gordon EM, Lee PS, Maisog JM, Foss-Feig J, Billington ME, VanMeter J, Vaidya CJ: Strength of default mode resting state connectivity relates to white matter integrity in children. Dev Sci 2011;14:738-751.

$>15$ Beaulieu C: The basis of anisotropic water diffusion in the nervous system - a technical review. NMR Biomed 2002;15:435-455.
16 Bennett IJ, Motes MA, Rao NK, Rypma B: White matter tract integrity predicts visual search performance in young and older adults. Neurobiol Aging 2012;33:433.e21e31.

17 Song SK, Sun SW, Ramsbottom MJ, Chang C, Russell J, Cross AH: Dysmyelination revealed through MRI as increased radial (but unchanged axial) diffusion of water. Neuroimage 2002;17:1429-1436.

18 Johnson NF, Kim C, Clasey JL, Bailey A, Gold BT: Cardiorespiratory fitness is positively correlated with cerebral white matter integrity in healthy seniors. Neuroimage 2012;59: 1514-1523.

19 Marks B, Katz L, Styner M, Smith J: Aerobic fitness and obesity: relationship to cerebral white matter integrity in the brain of active and sedentary older adults. Br J Sports Med 2011;45:1208-1215.

20 Marks B, Madden DJ, Bucur B, Provenzale JM, White LE, Cabeza R, Huettel SA: Role of aerobic fitness and aging on cerebral white matter integrity. Ann NY Acad Sci 2007; 1097: 171-174.

21 Voss MW, Heo S, Prakash RS, Erickson KI, Alves H, Chaddock L, Szabo AN, Mailey EL, Wójcicki TR, White SM, Gothe N, McAuley E, Sutton BP, Kramer AF: The influence of aerobic fitness on cerebral white matter integrity and cognitive function in older adults: results of a one-year exercise intervention. Hum Brain Mapp 2013;34:2972-2985.

$\checkmark 22$ Østby Y, Tamnes CK, Fjell AM, Walhovd KB: Morphometry and connectivity of the fronto-parietal verbal working memory network in development. Neuropsychologia 2011;49: 3854-3862. 
-23 Vestergaard M, Madsen KS, Baaré WF, Skimminge A, Ejersbo LR, Ramsøy TZ, Gerlach C, Akeson P, Paulson OB, Jernigan TL: White matter microstructure in superior longitudinal fasciculus associated with spatial working memory performance in children. J Cogn Neurosci 2010;23:2135-2146.

24 Lebel C, Walker L, Leemans A, Phillips L, Beaulieu C: Microstructural maturation of the human brain from childhood to adulthood. Neuroimage 2008;40:1044-1055.

25 Gebauer D, Fink A, Filippini N, JohansenBerg H, Reishofer G, Koschutnig K, Kargl R, Purgstaller C, Fazekas F, Enzinger C: Differences in integrity of white matter and changes with training in spelling impaired children: a diffusion tensor imaging study. Brain Struct Funct 2012;217:747-760.

26 Ogden CL, Kuczmarski RJ, Flegal KM, Mei Z, Guo S, Wei R, Grummer-Strawn LM, Curtin LR, Roche AF, Johnson CL: Centers for Disease Control and Prevention 2000 growth charts for the United States: improvements to the 1977 National Center for Health Statistics version. Pediatrics 2002;109:45-60.

27 Naglieri JA, Das JP: Cognitive Assessment System: Interpretive Handbook. Rolling Meadows, Riverside Publishing, 1997.
28 Gioia G, Isquith PK, Guy SC, Kenworthy L: Behavior rating inventory of executive function. Child Neuropsychol 2000;6:235-238.

29 Rorden C: DCM2NII (version October 7), 2007.

30 Smith SM, Jenkinson M, Woolrich MW Beckmann CF, Behrens TE, Johansen-Berg H, Bannister PR, De Luca M, Drobnjak I, Flitney DE, Niazy RK, Saunders J, Vickers J, Zhang Y, De Stefano N, Brady JM, Matthews PM: Advances in functional and structural MR image analysis and implementation as FSL. Neuroimage 2004;23:S208-S219.

31 Smith SM: Fast robust automated brain extraction. Hum Brain Mapp 2002;17:143-155.

32 Wakana S, Caprihan A, Panzenboeck MM, Fallon JH, Perry M, Gollub RL, Hua K, Zhang J, Jiang H, Dubey P, Blitz A, van Zijl P, Mori $S$ : Reproducibility of quantitative tractography methods applied to cerebral white matter. Neuroimage 2007;36:630-644.

33 Leemans A, Jeurissen B, Sijbers J, Jones DK: ExploreDTI: a graphical toolbox for processing, analyzing, and visualizing diffusion MR data. 17th Annu Meet Proc Int Soc Magn Reson Med, Honolulu, 2009, p 3536.

34 Klarborg B, Madsen K, Vestergaard M, Skimminge A, Jernigan TL, Baaré WF: Sustained attention is associated with right superior longitudinal fasciculus and superior parietal white matter microstructure in children. Hum Brain Mapp 2013;34:3216-3232.
5 Scholz J, Tomassini V, Johansen-Berg H: Individual differences in white matter microstructure in the healthy brain; in JohansenBerg H, Behrens TE (eds): Diffusion MRI: From Quantitative Measurement to in vivo Neuroanatomy. London, Elsevier, 2009, pp 105-126.

36 Beaulieu C: The biological basis of diffusion anisotropy; in Johansen-Berg H, Behrens TE (eds): Diffusion MRI: From Quantitative Measurement to in vivo Neuroanatomy. London, Elsevier, 2009, pp 105-126.

-37 Wheeler-Kingshott CAM, Cercignani M About 'axial' and 'radial' diffusivities. Magn Res Med 2009;61:1255-1260.

38 Ogden CL, Carroll MD, Kit BK, Flegal KM: Prevalence of obesity and trends in body mass index among US children and adolescents, 1999-2010. JAMA 2012;307:483-490.

39 McMurrer J: Instructional time in elementary schools: a closer look at changes for specific subjects [Internet]. Washington, DC: Center for Education Policy, 2008 [cited April 18, 2013]. (From the Capital to the Classroom: Year 5 of the No Child Left Behind Act). Available from: http://www.cep-dc.org/cfcontent_file.cfm?Attachment=McMurrer Report_InstructionalTime_022008.pdf. 